\title{
Visual Center of Gravity and Ratio of the Split Screen
}

\author{
By Yao Pirong \\ Yibin University's School of Computer \& Information Engineering \\ Yibin, China, 644000 \\ E-mail: 458468255@qq.com
}

\begin{abstract}
Divine Proportion has a dominant position in the picture composition; however, the picture perfect dichotomy screen out by its reasoning can not solve the massive distribution. In traditional quickly composition, due to the impact of 4:3 TV format, people are accustomed to the screen is divided into nine cells, because they are used by equipment manufacturers to provide the average split-screen "Wellshaped" dividing line; With the prevalence of 16:9 highdefinition TV and panoramic photography, photography cameraman shooting 16:9, this average segmentation approaches have failed to provide the composition with the help of. If you are still using the Divine Proportion different screen geometry segmentation, we can draw a dividing line position proportion that is consistent with the Divine Proportion in line with the physiological visual center of gravity of the human eye: 28:44:28; The new segmentation photography cameraman will provide a more convenient and quick reference picture composition.
\end{abstract}

Keywords- divine Proportion; rule of Thirds; location the visual center of gravity; the dividing line of the screen; position and scale

\section{The DOMINANCE OF Divine Proportion IMAGE} SCREEN

\section{A. Divine Proportion}

In ancient Greece in the 6th century $\mathrm{BC}$, the Pythagoreans found a line segment is divided into two parts, the part of (a) the ratio of the length $(a+b)$ is equal to another part (b) of this part ratio (a); namely

$$
\frac{a}{a+b}=\frac{b}{a}
$$

If it is assumed that $\mathrm{a}=1$, then:

$$
b^{2}+b-1=\sigma
$$

Solution of a quadratic equation known

$$
b=0.61803398875 \approx 0.618
$$

At the same time, the ancient Greek esthetician Plato deduced a "Divine Proportion" geometric formula from a square. Divided into square bottom edge bisection, take the center point $X$, from point $X$ to connect the adjacent corner $\mathrm{Y}, \mathrm{X}$ is the center of the circle, the line $\mathrm{XY}$ radius to draw a circle; The intersection of the circle with the bottom edge of a straight line to point $Z$, and extend the bottom edge to point $Z$, Thus, the square extends to a rectangle. Figure 1 [1].

If assuming a square of side length 1 , then:

$$
x y^{\prime}=0.5, y y^{\prime}=1
$$

$$
\begin{aligned}
& x y^{2}=x y^{\prime 2}+y y^{\prime 2}=0.5^{2}+1^{2}=1.25 \\
& \therefore x y=\sqrt{1.25}=1.11803398875 \approx 1.118 \\
& \therefore y^{\prime} z=x y-x y^{\prime}=1.118-0.5=0.618
\end{aligned}
$$

Thus obtaining a rectangular shape of an aspect ratio of $1: 1.618$ (Y point is the "golden point"):

$$
a: c=b: a=1: 1.618=0.618: \text { I }
$$

That is to say, in a rectangle than rectangular and square more in line with our visual habits, the most commonly used in people's daily necessities rectangular, deeper reason from the binocular vision of the human eye.

Later, after the majority of scholars and production practices found that the building or component of the design of the shape of the this proportion $(0.618: 1: 1.618)$, not only visually very beautiful soft and very durable solid, Therefore the 1:1.618 called the best ratio - of Divine Proportion. The Divine Proportion commonly used in the plastic arts, you can say that it has a dominant position in the aesthetic value of the plastic arts.

\section{B. Divine Proportion in picture composition - -picture perfect visual segmentation}

Figure 2: connected to the previously obtained having a rectangular upper left and lower right of the golden ratio, that is for the diagonal, and then from the upper right to the $\mathrm{Y}$ dots (Divine Proportion point) for a line segment intersects the diagonals, so put rectangle by Divine Proportion divided into three different parts; Using geometry we can very easily calculate the length of each of the three sides of three triangles still for 1.618:1:0.618.

If we want to show the scene along the lines of the three regions to arrange; visual habits will be accepted by the majority. This is called the most perfect rectangle dichotomy. In practical applications often figure horizontally or vertically flipped $180^{\circ}, 90$ and $270^{\circ}$; manner shown in Figure 3 will also be the smallest triangle turned upside down split.

\section{It does not solve all the screen visual problems that picture perfect visual segmentation}

We often have to face the screen may be inside the scene are not necessarily blocky distribution, such as the main body in the background above, in the background on the subject and accompany the body interacting with each other, etc. This time is not very good the massive split screen. Photography and painting or computer graphics rendering, often encounter this problem: If the main screen in the relatively small proportion, we should be placed to highlight what the position of the screen in order to maximize its

According to the Pythagorean Theorem that 
visual subjectivity? A large number of studies have shown that the screen can cause visual attention is not central, but the binocular vision the intersection of four points on the screen, these four points are referred to as "visual center of interest" or "visual focus".

So far, that position for visual center of interest the positioning method used in practice is the "average of three split": Trisection the length of two edges of the screen, it will screen is divided into nine squares with four lines; called "Jigging", four intersections formed by the four split line that is the best position for placing the main body [2].As shown in Figure 4.

\section{THE LOCATION OF VISUAL CENTER OF GRAVITY OF THE SCREEN}

\section{A. Binocular vision intersection to form a picture of the visual center of interest}

Why is the center of the screen can not cause visual attention, but most can cause visual attention to the four point of intersection formed by four dividing line? The visual physiology research found, each monocular visual range of an eccentric oval, monocular vision is this:

Right-eye visual range above 50 degrees and below 70 degrees, 60 degrees left and right 100 degrees, photopic center axis is not in the central $50 \%$, but biased to the right of the $60 \%$; Similarly, the left eye is above 50 degrees and below 70 degrees, the right 60 degrees, 100 degrees left photopic center axis is not in the central $50 \%$ but $60 \%$ of the partial right [3]. In $25 \mathrm{~cm}$ in photopic distances, this each eccentric oval visual intersection with the center axis of the other photopic and formed four intersections, these four points, since the same time by two eye photopic axis attention, so they become the visual center of gravity of the screen. As shown in Figure 5.

In Figure 5, the left eye of the visual range is represented with a red circle, the circle is not perfect circular or elliptical, but half of the composition consists of two elliptical depicting them up and down the same radius about different radii (ratio 3:5), the visible range of the right eye with a green circle represents, if the screen is bright distance, two at the same time watch the visual range of the yellow circle represents, two eye vision is the same, the yellow ellipse is symmetrical labyrinth ratio of 10:6 elliptical.

In such a binocular intersection formed within the visual range, each place are not visually sensitive areas, or visual sensitivity in each region is different; The most sensitive areas are the intersection point, that the intersection point of the vision of the left eye view and right visual center axis, and the center axis of the vision of the right eye and the left visual intersection. This is the visual center of gravity of the screen physiology principle, some scholars turn visual center of gravity of the screen called the visual center of interest.

\section{B. This position of the visual center of gravity Physiology} can take advantage of the golden section method to get it?

Visually seen from Figure 5, the two positions are inconsistent, eyes intersection of the visual center of gravity and the traditional average trichotomy figure obtained by dividing the intersection of four lines. This trichotomy is only approximate and not precise split screen method, especially for the recently popular 16:9 format, if you use the Rule of Thirds composition and body placed in the intersection of four dividing line, it is too near to the screen central.

Can not take advantage of the Divine Proportion plus geometry resulting from the visual center of gravity position? And this position is consistent with the binocular vision of human physiology and consistent with the classic Divine Proportion.

Let us first or 1:1.618 Divine Proportion rectangle split screen: As shown in Figure 6.

The four edges of the screen ratio of 1:1.618 the find the Divine dividing point $\mathrm{Y}$, a total of eight such Divine split point; Divine split point and the edge of the adjacent vertices connected by line segments. In the figure, we can clearly see that the eight lines and two diagonal form four intersection points; the four intersection of the visual center of gravity very close for the two visual maps out the terms of physiology; This Description reasoning the Divine Proportion applies to the center of gravity of binocular vision.

We are the most commonly used format than: $4: 3,3: 2$, and 16:9, 3:2 frame closest on Figure 1.618:1 frame digital SLR camera. If we put the above Figure 5 and Figure 6 with a 16:9 aspect ratio to redraw what the results of it? The results are shown in Figure 7:

From the chart we can visually be seen, both the visual center of gravity position is very close; so we have reason to believe, Divine Proportion applies not only to the screen scene was massive distribution screen, also applies to the subject in the background above, the main and interacting with each other in the background on the screen to accompany the body.

\section{THE IDEAL RATIO OF SPLIT SCREEN}

\section{A. The ideal scale model split screen}

Let us with a straight line connecting these four visual focus use the Divine Proportion reasoning out, and to extend it to intersect with the four edges of the screen, so that the same screen is divided into nine squares. It is different with the now popular trisection average proportion of split, is obtained through mathematical calculations; function equation illustrated in Figure 8 shows.

Coordinate origin is in the lower left corner of the screen, the screen itself is 100:161.8 best ratio rectangle, then its coordinates is $(61.8,100)$ that the left side of the rectangular upper gold point, it with the coordinate origin of the linear equation:

$$
y=1.618 x
$$

While the upper-left corner of the connection screen with a diagonal line in the lower right corner of the equation as follow:

$y=100-0.618 x$

Solution of two linear equations in two available:

$$
x=44.72, y=72.4
$$


Therefore, it coordinates $(44.72 ; 72.4)$ that the visual center of gravity in the upper left of the screen. Using the same method, we can calculate the coordinates of the three additional visual focuses, and so will be able to calculate the position of the dividing line and the split ratio:

$44.72 \div 161.8=0.2764 \approx 28 \%$.

Namely, the aspect ratio of 1.618:1 of the screen dividing the optimum ratio is not each part is the average ratio of $33.3 \%$, but the proportion of such press $28: 44: 28$ to split.

So for now the most popular $16: 9$ and $3: 2$, this ratio? With the same geometric figures can be proved that the proportion of the dividing line position still is $28: 44: 28$. Figure 9:

\section{B. Application of the ideal screen division line in reality}

Order more convenient for users to take the picture composition viewfinder, the vast majority of digital cameras and digital camera viewfinder provides a screen dividing line, commonly known as the grid lines. However, due to various reasons, the use of split screen lines are trichotomy evenly distributed. We can easily be seen from Figure 9, the smaller the ratio of the length and width of the screen, the location of the visual center of gravity of the closer position on average segmentation method. Traditional television screen is the length and width of a relatively small proportion of 4:3 frame, so when the camera using a the average the trichotomy split screen, this deviation is not too large that the use of the average dividing line to get the center of gravity of the screen with ideal visual location of the center of gravity.
However, in recent years with the popularity of the screen of 16:9 high-definition TV screen and panoramic photography, many photographers and cameramen began to feel in the camera or camcorder viewfinder window dividing line can not provide help in the composition. They feel their eyes visual center and the center of gravity of the screen this average dividing line does not overlap, If you press the camera or camcorder viewfinder the window dividing line placed in the main, shooting out of the picture will make people think ugly.

After a long practice found that now the average dividing line in the viewfinder window has been unable to meet in the now popular wide screen composition, on its deeper reason is that prior to this we do not by Divine Proportion precise positioning the geometric position of the parting line of the screen.

The study will be imaging equipment manufacturers provide reference; When they designed viewfinder window dividing line, if at 28:44:28 the proportion of distribution of the dividing line, will help equipment users quickly composition viewfinder shooting.

\section{REFERENCES}

[1] Yao Pei rong. More on photographic composition [J], Silicon Valley, on May 2008

[2] Yan Zhigang. Photography art tutorial [M], p. 106, Fudan University Press, sixth edition in November 2010.

[3] JierEnjiang. Grazier astronomicalforum,[EB/OL].[2008-8-1]. http://www.astronomy.com.cn.

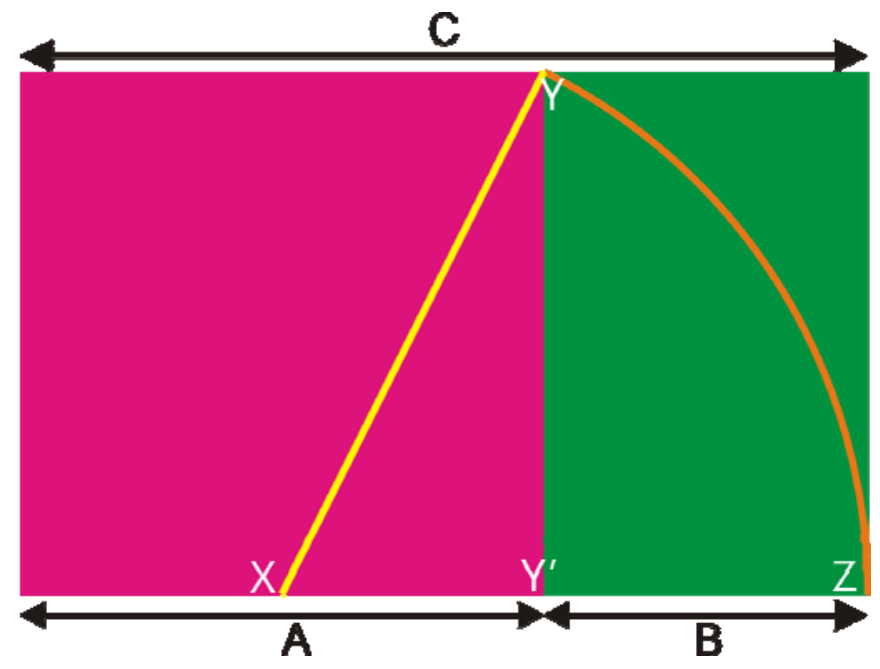

Figure 1:The Divine Proportion Plato geometric schematic. 

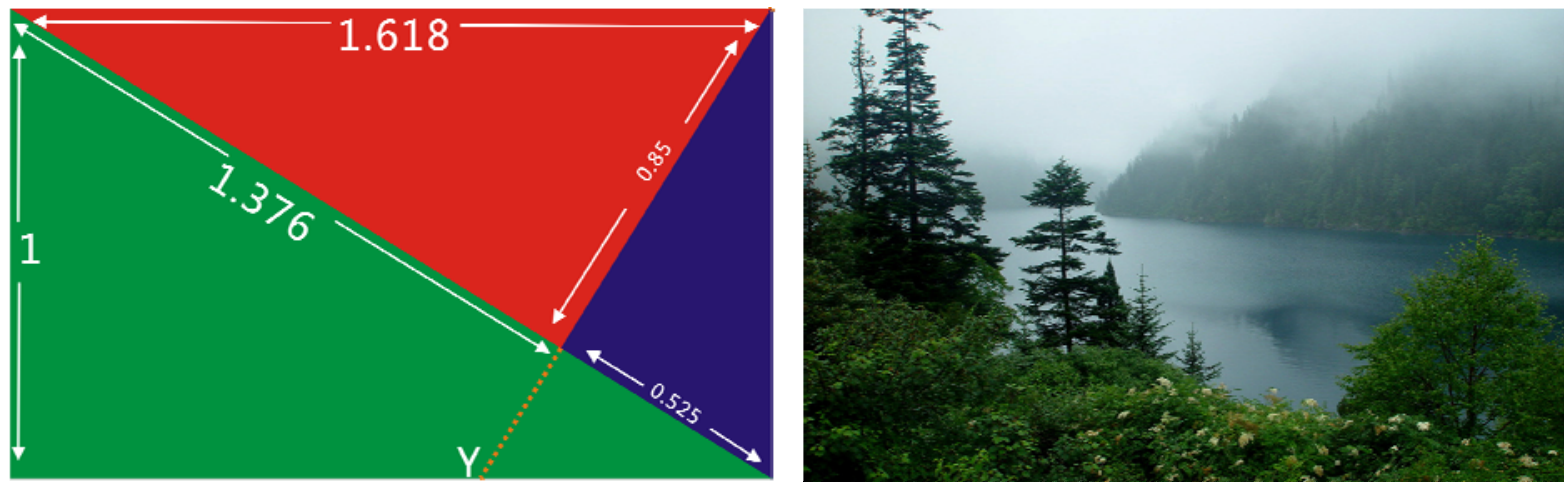

Figure 2: The horizontal picture perfect trichotomy split screen icon
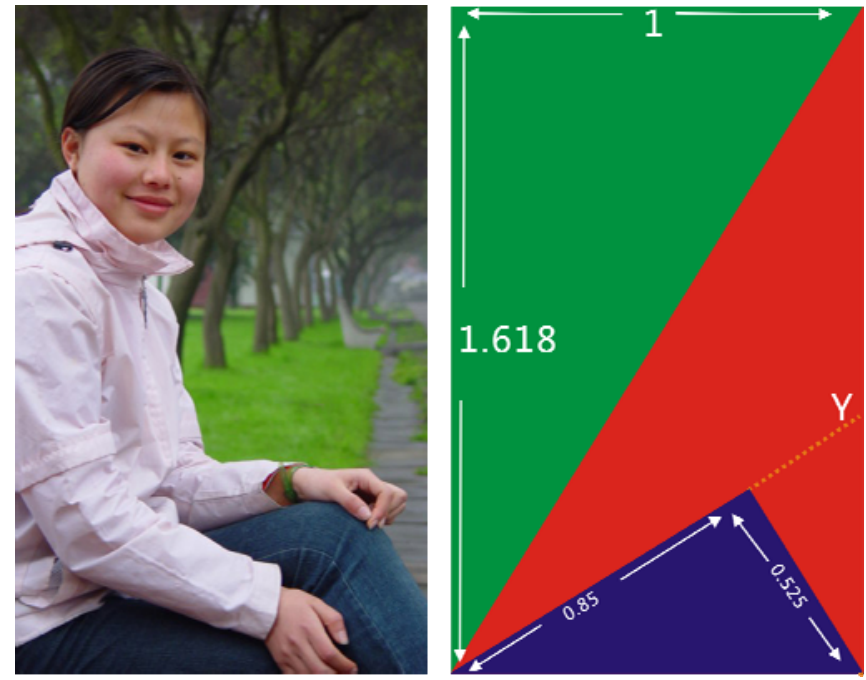

Figure 3: The vertical picture perfect trichotomy split screen icon

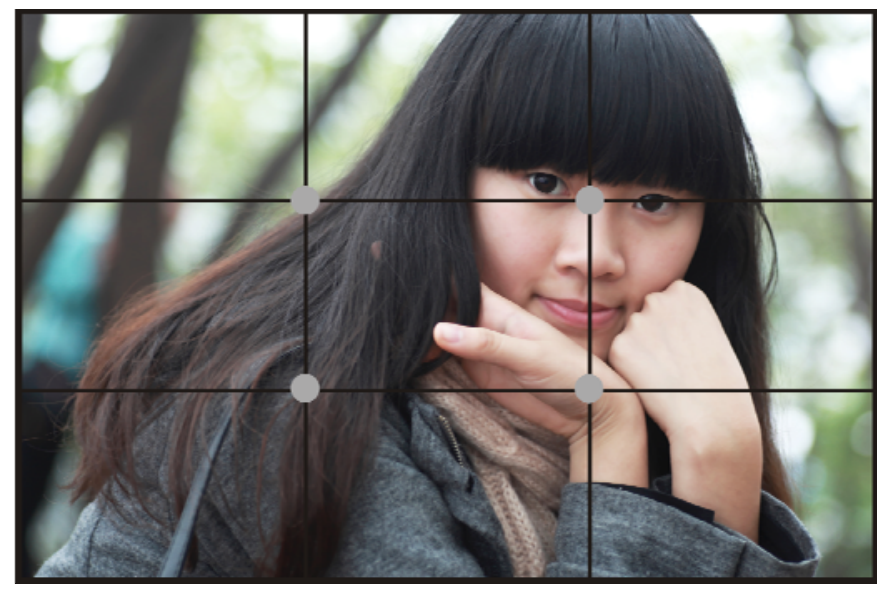

Figure 4: Trisection screen dividing the position of the center of gravity of the screen 

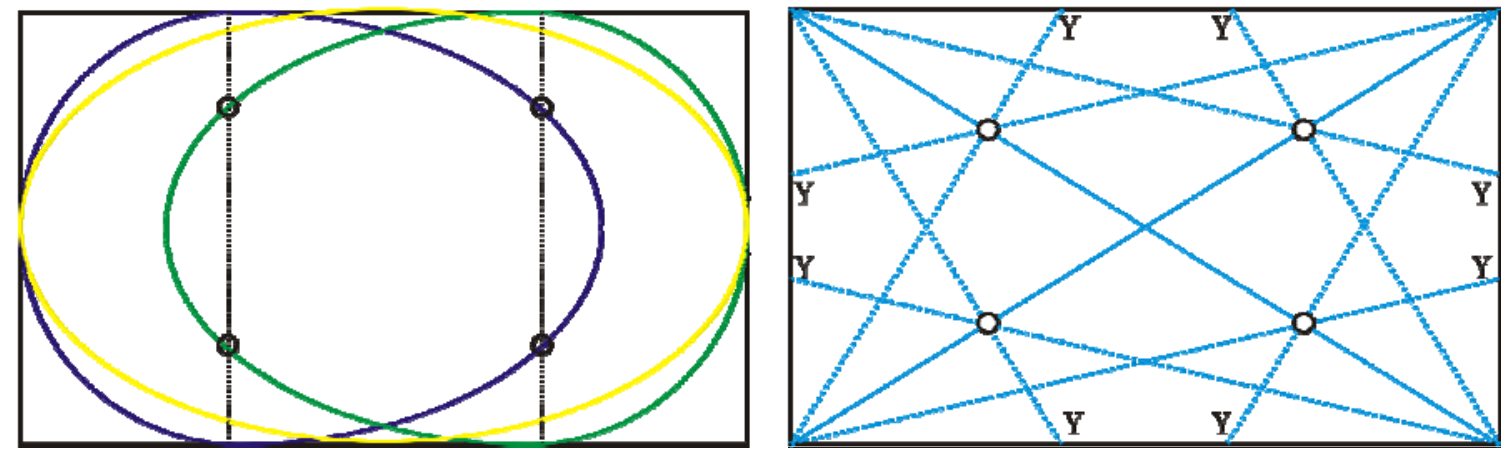

Figure 5: Human binocular vision range intersection icon

Figure 6: Use the Divine Proportion draw visual center of gravity position

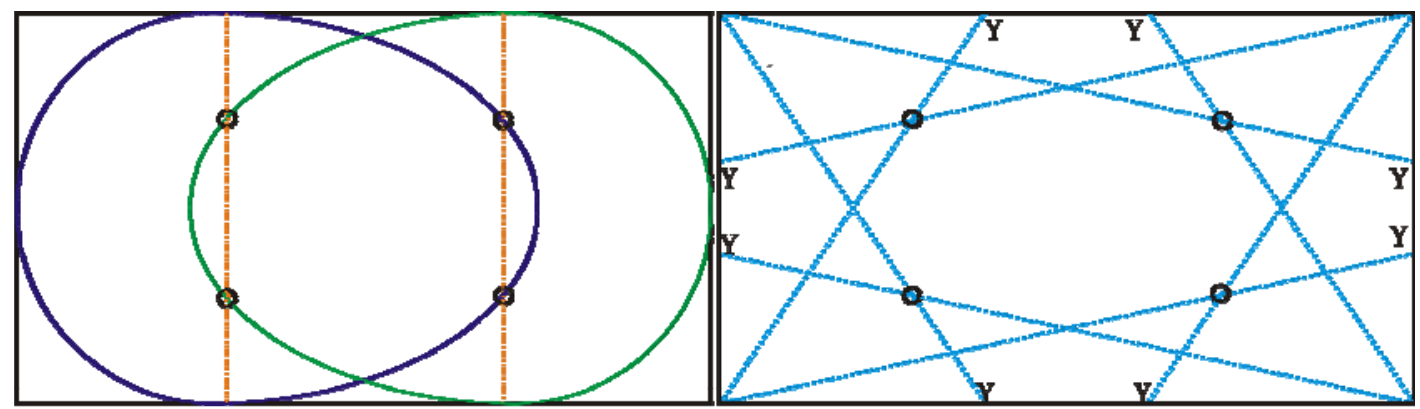

Figure 7: Use the Divine Proportion drawn16:9 screen visual center of gravity position

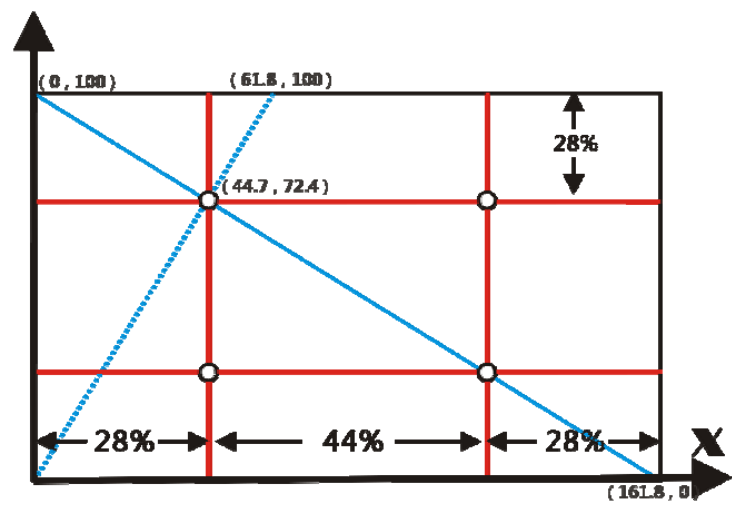

Figure 8: Visual center of gravity position geometry solving equations icon
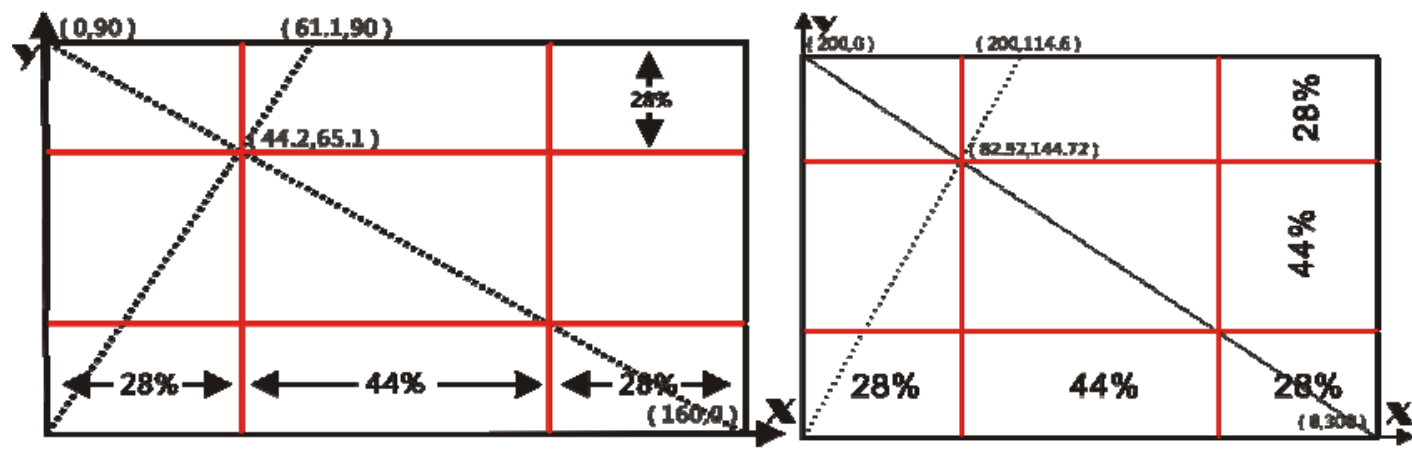

Figure 9: $16: 9$ and 3:2 split ratio screen icon 